\title{
Atividade elétrica muscular em paciente com Ataxia de Friedreich - estudo de caso
}

\author{
Electrical muscle activity in a patient \\ with ataxia Friedreich - case study
}

FisiSenectus . Unochapecó Ano 3, n. 1 - Jan/Jun. 2015 p. $11-17$

Iassana Biasi. iassana.biasi@gmail.com

Fisioterapeuta graduada pela Universidade Comunitária da Região de Chapecó Unochapecó.

Miriane Gava. miri_gava007@hotmail.com

Fisioterapeuta graduada pela Universidade Comunitária da Região de Chapecó -

Unochapecó.

Josilene S. Conceição Kaminski. josilene.kaminski@unoesc.edu.br

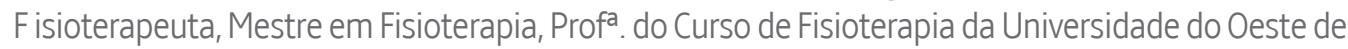
Santa Catarina (Unoesc).

Franciane Barbieri Fiório. franciane.fiorio@unoesc.edu.br

Fisioterapeuta, Doutora em Ciências da Reabilitação, Profá. do Curso de Fisioterapia da Universidade do Oeste de Santa Catarina (Unoesc).

\section{Resumo}

Introdução: a Ataxia de Friedreich é uma doença neurodegenerativa progressiva, de herança autossômica recessiva e com início precoce. A manifestação predominante é a incoordenação motora ocasionada devido ao acometimento do cerebelo. Objetivos: este estudo tem como objetivo verificar se há diferença na atividade elétrica muscular do deltoide e tríceps braquial de um paciente com Ataxia de Friedreich durante a realização de flexão do ombro, quando o exercício é realizado com facilitação e com ou sem consciência corporal. Materiais e métodos: o estudo foi realizado com um paciente de 61 anos com diagnóstico clínico de Ataxia de Friedreich. Realizou-se a análise da atividade eletromiográfica dos músculos deltoide e tríceps braquial durante a realização da flexão de ombro em três diferentes formas (com facilitação pelo fisioterapeuta e com e sem consciência corporal). Resultados: houve maior magnitude da atividade elétrica muscular nos músculos deltóide e tríceps braquial durante a tarefa de flexão do ombro realizada sem consciência corporal em comparação com as outras formas de realização. Conclusão: conclui-se que há diferença na atividade elétrica muscular do deltoide e tríceps braquial de acordo com a tarefa realizada. A magnitude da atividade elétrica é maior no movimento de flexão sem consciência corporal, porém os movimentos realizados com consciência corporal e com facilitação foram mais coordenados.

\section{Palavras-chave}

Ataxia de Friedreich, fisioterapia, eletromiografia.

\section{Fisiß̌nectus}




\begin{abstract}
Introduction: Friedreich's Ataxia is a progressive neurodegenerative disease, autosomal recessive, and with early onset. The predominant manifestation is incoordination caused due to the involvement of the cerebellum. Objectives: this study aims to observe the differences in muscle electrical activity of the deltoid and triceps of a patient with Friedreich's Ataxia, while performing shoulder flexion when the exercise is performed with facilitation and with and without body awareness. Materials and Methods: the study was conducted with 61 patients with clinical diagnosis of Friedreich ataxia. We conducted analysis of the electromyographic activity of the deltoid muscles and triceps during the course of shoulder flexion in three different forms (with facilitation by the physiotherapist, and with and without body awareness). Results: there was a greater magnitude of muscle electrical activity in the deltoid and triceps during shoulder flexion task performed without body awareness compared to other embodiments. Conclusion: it concludes that there is a difference in muscle electrical activity of the deltoid and triceps according to the task performed. The magnitude of the electrical activity is greater in flexion without body awareness, but the movements performed with body awareness and facilitation were more coordinated.
\end{abstract}

\title{
Keywords
}

Friedreich ataxia, physical therapy specialty, electromyography.

\section{Introdução}

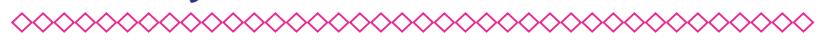

A Ataxia de Friedreich, descrita pela primeira vez em 1863 por Nicholaus Friedreich, é uma doença neurodegenerativa progressiva, de herança autossômica recessiva, de início precoce e caracterizada pela incoordenação motora devido ao envolvimento do cerebelo ${ }^{1}$. Apresenta etiologia desconhecida, sem cura ou tratamento etiológico e consiste na doença mais comum dentre as hereditárias, tendo uma prevalência de aproximadamente 1:50.000 2,3.

O cerebelo é responsável pelo equilíbrio, pelo controle das atividades musculares rápidas, pela coordenação, pela correção e pelo aprendizado motor. O comprometimento dessa área pode causar a ausência da coordenação motora observada por meio de déficits na velocidade, amplitude de movimento e força muscular, além de alterações no planejamento e aprendizado motor ${ }^{4}$.

A manifestação clínica da Ataxia de Friedreich, em geral, ocorre entre a primeira ou segunda década de vida, caracterizada por ataxia lentamente progressiva, que se inicia, geralmente, nos membros inferiores e, posteriormente, comprometendo o tronco e os membros superiores. Também observa-se a ausência de reflexos profundos, disartria, nistagmo, sinais de Babinski e perda de sensibilidade em fases mais tardias da doença. Uma característica importante é a hipotonia muscular que ocasiona outros comprometimentos como, tremor, fenômeno do rechaço, disdiadococinesia, dissinergia e dismetria que levam ao comprometimento da deambulação e das atividades de vida diária ${ }^{\mathbf{1 , 5 , 6}}$.

Diante das perdas funcionais é de suma importância que o paciente tenha acompanhamento fisioterapêutico, visando o treino dos movimentos nos quais apresenta dificuldade, interferindo com movimento controlado e efetivando a conquista de metas e possibilitando, assim, melhores habilidades na realização de tarefas, que, muitas vezes, tornam-se dispendiosas devido ao grande gasto energético provocado pela incoordenação dos movimentos ${ }^{7,8}$.

Quando o paciente realiza os movimentos aleatoriamente, mesmo que funcionais, estes são incoordenados. Nesse caso, a intervenção do fisioterapeuta consiste, basicamente, em dois princípios: a facilitação do movimento desejado, o qual é realizado pelo paciente com auxílio do fisioterapeuta que guia o movimento do paciente; e a consciência corporal que consiste 
no aprendizado da realização do movimento, ou seja, o paciente deve prestar atenção no movimento durante sua execução, o que o torna mais harmônico.

Nesse sentido, este estudo tem como objetivo verificar se há diferença na ativação muscular quando o exercício é realizado com facilitação e com ou sem consciência corporal em pacientes com Ataxia de Friedreich, o que pode subsidiar o trabalho do fisioterapeuta.

\section{Materiais e métodos}

$\infty \times \infty \times \infty \times \infty \times \infty \times \infty \times \infty \times \infty \times \infty \times \infty \times \infty \times \infty \times \infty$

Este estudo foi desenvolvido na Universidade Comunitária Regional de Chapecó (Unochapecó), sendo aprovado pelo Comitê de Ética sob o protocolo de número 356/2012. O participante assinou o termo de consentimento livre e esclarecido e concordou em participar da pesquisa de forma voluntária.

Trata-se de um estudo de caso realizado com um paciente de 61 anos com diagnóstico clínico de Ataxia de Friedreich. 0 paciente relatou que aos 35 anos observou redução de força em membros inferiores, sofrendo quedas com certa frequência, no entanto, só procurou assistência médica após três anos do início dos sintomas. Os sintomas da doença evoluíram com o decorrer dos anos e aos 47 anos o paciente passou a depender de cadeira de rodas para locomoção. Ele apresenta, também, dificuldade na realização das atividades de vida diária devido à incoordenação dos movimentos em membros superiores e inferiores. Por outro lado, o paciente não apresenta cardiopatia ou diabetes, doenças comuns em pacientes atáxicos.

Para aquisição e monitoramento da atividade elétrica muscular foi utilizado um eletromiógrafo de superfície da marca Miotec ${ }^{\circledR}$, modelo Miotool 400 de 4 canais. 0 sinal do eletromiógrafo foi coletado usando eletrodos superficiais bipolares de $\mathrm{Ag} / \mathrm{AgCl}$, circulares, com área condutiva de 1 $\mathrm{cm}$ de diâmetro e distância intraeletrodos centro a centro de $20 \mathrm{~mm}$ (Meditrace $®$ pediátrico).

Para o posicionamento dos eletrodos nos músculos deltóide (fibras anteriores) e tríceps braquial do membro superior direito, seguiram- se os procedimentos recomendados pela Seniam (Surface Eletromyography for the non-invasive Assessment of muscle). No local de colocação do eletrodo foi realizada assepsia com algodão umedecido em álcool $70 \%$ e tricotomização, quando necessário.

A escolha desses músculos foi motivada devido à atividade funcional que o paciente realizou, visto que este permaneceu na postura sentada, com os pés apoiados no solo, sem apoio posterior e com as mãos sobre as coxas. Partindo dessa posição, com o membro superior direito o paciente realizou flexão de ombro até $90^{\circ}$, com extensão de cotovelo para alcance de um objeto oferecido à sua frente. A altura e distância entre o objeto e o paciente foram padronizadas para todas as coletas.

Essa atividade funcional, de alcance do objeto, foi realizada de três diferentes formas, sendo que na primeira foi solicitado que paciente pegasse o objeto sem dar-Ihe muitas informações, permitindo, assim, que ele agisse de maneira usual. Na segunda, o paciente realizou a atividade funcional de forma facilitada pelo pesquisador, seguindo os princípios do Conceito Neuroevolutivo Bobath, em ponto-chave cotovelo. Na terceira forma o paciente realizou a atividade funcional seguindo o princípio da realização do movimento com consciência corporal, no qual o movimento é realizado lentamente para que o paciente consiga ter a percepção corporal do movimento, além de acompanhá-lo com os olhos, obtendo assim um feedback visual. Em cada forma de realização do movimento foram realizadas cinco tentativas para captura do sinal eletromiográfico, dando ao paciente um minuto de repouso entre uma tomada e outra, para evitar fadiga muscular.

Antes do início da aquisição dos sinais, três tentativas foram realizadas com o objetivo da familiarização do paciente com as tarefas propostas. Os participantes foram solicitados a informar (por meio da palavra "ok") quando fosse possível iniciar a coleta dos dados. Além disso, o paciente foi requerido a não praticar atividade física, nem a fazer qualquer exercício estressante 24 horas antes da coleta. Cabe destacar que todos os procedimentos descritos foram executados pelos mesmos pesquisadores. 
Após a coleta do sinal eletromiográfico, os dados brutos analógicos foram janelados e filtrados (filtro passa-banda de $20 \mathrm{~Hz}$ a $500 \mathrm{~Hz}$ ). 0 janelamento foi realizado durante o tempo de contração muscular, desde o início até o final da atividade de alcance, que variou de uma forma de realização do movimento para outra.

Em seguida à coleta e à avaliação qualitativa dos sinais mioelétricos, optou-se pela quantificação das fases de ativação dos músculos com relação à amplitude, realizada por meio do valor RMS (Root Mean Square) $(\mu v)$, que representa a raiz quadrada da média dos quadrados de corrente ou da voltagem ao longo do ciclo. Tal escolha justifica-se por este ser o parâmetro que melhor reflete o grau de atividade elétrica muscular 9 .

Os dados foram tabulados usando o software Microsoft Excel2007, e a verificação da normalidade da distribuição das variáveis foi realizada por meio do teste de Shapiro-Wilk. Como os dados apresentaram distribuição normal, foi utilizado ANOVA com teste de Turkey's post hoc para comparação das médias do RMS $(\mu \mathrm{V})$ nas diferentes formas de realização da atividade funcional. Cabe mencionar que os dados foram expressos com valor das médias da atividade elétrica múscular e foi utilizado o programa estatístico Bioestat $5.0 ®$ com a adoção do nível de significância $p<0,05$.

\section{Resultados}

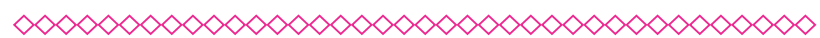

Os valores médios da variável eletromiográfica Root Mean Square (RMS) em microvolt $(\mu \mathrm{V})$ são mostrados na Tabela $\mathbf{1}$. Houve maior atividade elétrica muscular do músculo deltóide durante a atividade de alcance sem consciência corporal, quando comparado com as demais formas de realização dessa tarefa.

A diferença estatisticamente significativa ocorreu entre a atividade realizada sem consciência corporal e com facilitação $(p<0,05)$. Para o músculo tríceps braquial, também houve maior atividade elétrica muscular quando a atividade de alcance foi realizada sem consciência corporal, tal diferença ocorreu quando comparada à atividade realizada com consciência corporal $(p<0,01)$. A diferença estatisticamente significativa entre as atividades de alcance realizadas com facilitação e com consciência corporal $(p<0,05)$ também ocorreram.

Os valores médios $(\mu \mathrm{V})$ da atividade elétrica muscular do deltoide e tríceps braquial nas três diferentes formas de realização do exercício estão representadas no Gráfico 1 . Observa-se que na atividade realizada sem consciência corporal a atividade elétrica do músculo deltóide foi maior que do músculo tríceps, com uma diferença de 193,4 $\mu \mathrm{V}$. Na atividade realizada com consciência corporal a atividade elétrica do deltóide também foi maior que do tríceps braquial, com uma diferença de $136,4 \mu \mathrm{V}$. Na atividade com facilitação o músculo deltóide também apresentou maior atividade elétrica do que o tríceps braquial, no entanto, a diferença entre a atividade elétrica foi pequena, de $18,6 \mu \mathrm{V}$.

\section{Discussão}

$\infty \times \infty \times \infty \times \infty \times \infty \times \infty \times \infty \times \infty \times \infty \times \infty \times \infty \times \infty \times \infty \infty)$

Ao longo da análise do sinal eletromiográfico durante a tarefa de flexão do ombro observouse uma maior atividade elétrica muscular nos músculos deltóide e tríceps braquial na execução sem consciência corporal, ou seja, quando o paciente realizou o movimento aleatoriamente (sem interferência do fisioterapeuta). No entanto, observa-se que houve uma maior variação na oscilação dos picos (máximos e mínimos) durante essa atividade. Isso pode ser explicado devido ao fato dos movimentos serem incoordenados em pacientes com Ataxia de Friedreich.

Já nas atividades realizadas com consciência e com facilitação, essa variação de picos foi menor, podendo ser associada a uma execução do movimento mais harmonioso, com menos ataxia e, consequente, menor atividade elétrica muscular. Sugere-se que a facilitação para a realização da tarefa proposta favoreceu a menor atividade desses músculos, sendo que, apesar do desempenho da mesma função, a interferência do fisioterapeuta pode ser considerada benéfica, pois a menor atividade elétrica muscular gera menor gasto energético. 
Outro estudo mostrou que os exercícios parecem modular o sistema de controle postural e diminuir a atividade elétrica muscular, porém esses resultados ainda estão em debate. Tal estudo investigou o efeito da prática de exercício físico sobre a atividade elétrica (ajustes posturais antecipatórios) em idosos em uma tarefa que envolvia o levantamento de uma carga com os braços.

Este estudo evidenciou que idosos praticantes de exercícios físicos apresentaram uma menor atividade eletromiográfica do músculo tibial anterior quando comparados a idosos sedentários, sugerindo, assim, que o exercício pode alterar as estratégias de controle postural $^{10}$.

Estudos neurológicos por imagem têm confirmado a ideia de que o cerebelo entra em atividade em processos cognitivos como os de organização espacial, visual e de memória de movimento tanto em curto quanto em longo prazo $^{4}$. Nesse sentido, as atividades realizadas com consciência corporal e com facilitação estimularam as percepções proprioceptivas, acarretando em um movimento menos incoordenado.

Durante o processo de aquisição de uma habilidade motora, o córtex é totalmente exigido enquanto o processo de aquisição se consolida. Após a automatização, os movimentos passam a ocorrer na área subcortical (núcleos da base, cerebelo e tálamo) ${ }^{11,12}$. Na Ataxia de Friedreich, onde há lesão no cerebelo, essa automatização é prejudicada, com isso, o paciente necessita lançar mão de recursos somatossensoriais para realizar os movimentos com menos incoordenação.

$\mathrm{Na}$ execução da tarefa de alcance com consciência corporal, onde o paciente realizou a atividade com recursos intencionais conscientes (visão e propriocepção consciente) e na atividade com facilitação, na qual o terapeuta provê o paciente com alguns dos recursos proprioceptivos, houve uma melhora na parametrização do movimento, tornando-o mais lento, porém com menor amplitude, ou seja, menos oscilação, menos erros de direção ao alvo, com consequentes movimentos mais coordenados.

Sem dúvida, este estudo apresenta limitações, como a participação de apenas um sujeito. Por outro lado, investigando a atividade elétrica de tais músculos em três tarefas funcionais, foi possível identificar modificações nos parâmetros eletromiográficos que favoreceram novas pesquisas.

\section{Conclusão}

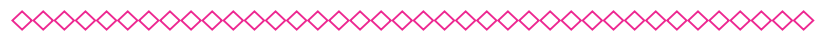

Os resultados deste estudo permitem concluir que quando os exercícios são realizados com consciência corporal e com facilitação pelo fisioterapeuta os movimentos tornam-se mais coordenados e com parametrização menos discrepante do movimento normal, facilitando, assim, uma atividade muscular mais efetiva dos grupos específicos para a realização da atividade de alcance.

Devido a isso, salienta-se a grande importância do tratamento fisioterapêutico que irá reeducar o paciente, proporcionando a execução de movimentos mais harmônicos e com menos gasto energético, visando atividades funcionais mais eficazes. Contudo, são necessários novos estudos das técnicas de fisioterapia na efetivação da atividade muscular de pacientes com Ataxia de Friedreich, principalmente considerando uma amostra maior.

\section{Referências}

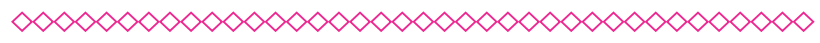

1. Rinaldi C, Tucci T, Maione S, Giunta A, De Michele G, Filla A. Low-dose idebenone treatment in Friedreich's ataxia with and without cardiac hypertrophy. J Neurol, 2009;256(9):1434-1437.

2. Pandolfo M. Friedreich ataxia: The clinical Picture. J Neurol. 2009; 256 [Suppl 1]:3-8.

3. Martelli A, Friedman LS, Reutenauer L, Messadeq N, Perlman SL, Lynch DR et al. Clinical data and characterization of the liver conditional mouse model exclude neoplasia as a non- 
neurological manifestation associated with

Friedreich's ataxia. Dis. Model Mech.. 2012;5(6):

860-869

4. Gordon N. The cerebellum and cognition. Eur J Pediat Neurol. 2007 Jul;11(4): 232-234.

5. Delatycki MB. Evaluating the progression of Friedreich ataxia and its treatment. J. Neurol.2009;256[suppl.1]:36-41.

6. Cardozo DC, Seixas FAV. Ataxia de Friedreich: relato de um caso com manifestação tardia. Arq. Ciênc. Saúde.2009;13(3):243-247.

7. Silveira BT, Grave MTQ, Périco E. Avaliação da força muscular e amplitude de movimento em paciente portador de ataxia de friedreich: um estudo de caso. Caderno pedagógico. 2012;9(2):49-58

8. Torriani-Pasin C, Arjona M, Leitão RG, Lima RZ, Cyrillo FN. Efeitos da dupla tarefa na marcha de pacientes atáxicos. Science in Health. São Paulo mai-ago 2010; 1(2):101-110.

9. Balasubramanian CK, Bowden MG, Neptune RR, Kautz SA. Relationship between step length asymmetry and walking performance in subjects with chronic hemiparesis. Arch. Phys. Med.

Rehabil. 2007 Jan; 88(1):43-49.

10. Carvalho R, Vasconcelos $O$, Gonçalves $P$, Conceição F, Vilas-Boas JP. The effects of physical activity in the anticipatory postural adjustments in elderly people. Motor Control. 2010;14(3):371-9.

11. Bond JM, Morris ME. Goal-directed secondary motor tasks: their effects on gait in subjects with Parkinson's disease. Arch. Phys. Med. Rehabil. 2000 Jan; 81(1): 110-614.

12. Cookburn J, Haggard P, Cook J, Fordham C. Changing patterns $f$ cognitive-motor interference (CMI) over time during ecovery from stroke. Clin. Rehabil. 2003 Mar;17(2):167-73. 


\section{Anexos}

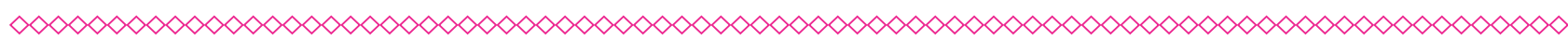

Tabela 1 - Valores médios da variável eletromiográfica Root Mean Square (RMS) em microvolt $(\mu \mathrm{V})$

\begin{tabular}{|l|c|c|c|c|}
\hline \multicolumn{1}{|c|}{ Domínios } & Deltoide & Valor de $\mathbf{p}$ & $\begin{array}{c}\text { Tríceps } \\
\text { Braquial }\end{array}$ & Valor de $p$ \\
\hline Sem consciência & 852,0 & & 658,6 & \\
\hline Com consciência & & & & $<0,01$ \\
\hline & 744,0 & $<0,05$ & 607,6 & \\
\hline Com facilitação & & & & $<0,05$ \\
\hline
\end{tabular}

(clique para voltar ao texto)

Gráfico 1 - Valores médios $(\mu \mathrm{V})$ da ativação muscular de deltoide e tríceps braquial nas três diferentes formas de realização do exercício.

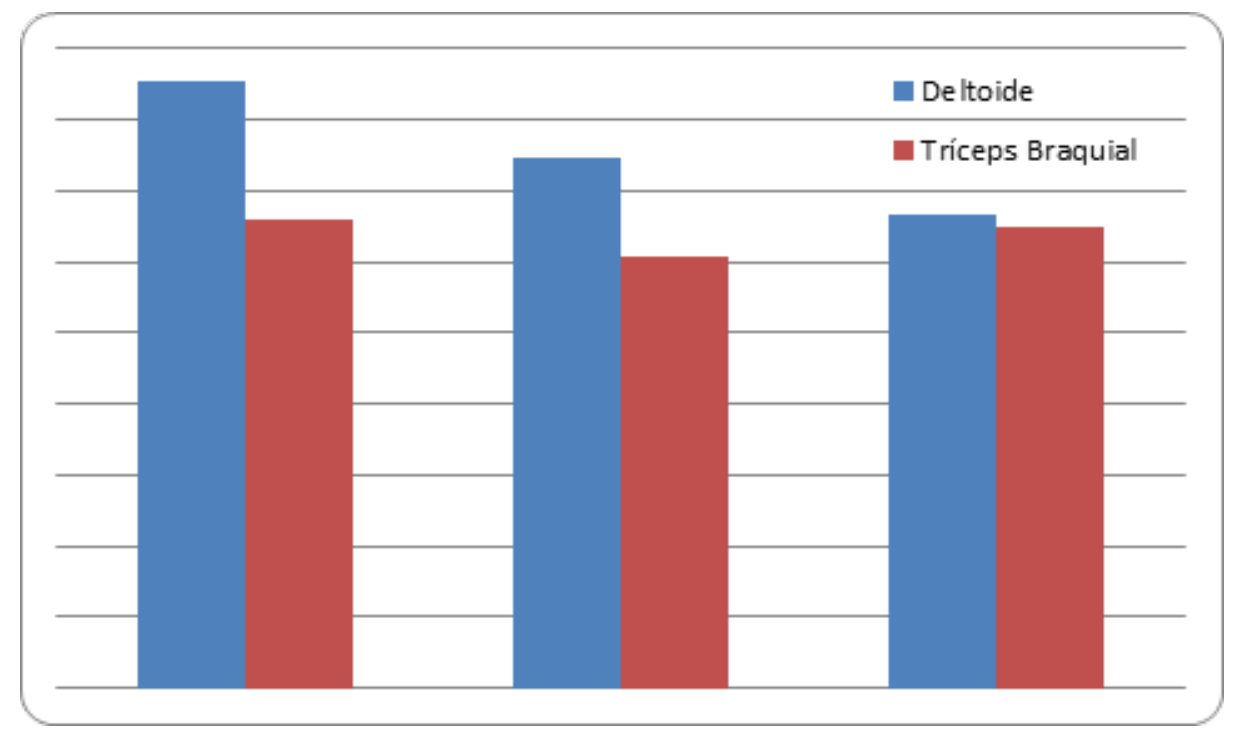

(clique para voltar ao texto) 